This copy is for your personal, non-commercial use only.

If you wish to distribute this article to others, you can order high-quality copies for your colleagues, clients, or customers by clicking here.

Permission to republish or repurpose articles or portions of articles can be obtained by following the guidelines here.

The following resources related to this article are available online at www.sciencemag.org (this information is current as of October 28, 2010 ):

Updated information and services, including high-resolution figures, can be found in the online version of this article at:

http://www.sciencemag.org/cgi/content/full/328/5979/749

Supporting Online Material can be found at:

http://www.sciencemag.org/cgi/content/full/science.1185837/DC1

A list of selected additional articles on the Science Web sites related to this article can be found at:

http://www.sciencemag.org/cgi/content/full/328/5979/749\#related-content

This article cites 27 articles, 7 of which can be accessed for free:

http://www.sciencemag.org/cgi/content/full/328/5979/749\#otherarticles

This article has been cited by 1 article(s) on the ISI Web of Science.

This article has been cited by 2 articles hosted by HighWire Press; see:

http://www.sciencemag.org/cgi/content/full/328/5979/749\#otherarticles

This article appears in the following subject collections:

Immunology

http://www.sciencemag.org/cgi/collection/immunology 


\section{Induction of Lymphoidlike Stroma and Immune Escape by Tumors That Express the Chemokine CCL21}

Jacqueline D. Shields, ${ }^{*}$ Iraklis C. Kourtis, ${ }^{*}$ Alice A. Tomei, Joanna M. Roberts, Melody A. Swartz†

Tumor manipulation of host immunity is important for tumor survival and invasion. Many cancers secrete CCL21, a chemoattractant for various leukocytes and lymphoid tissue inducer cells, which drive lymphoid neogenesis. CCL21 expression by melanoma tumors in mice was associated with an immunotolerant microenvironment, which included the induction of lymphoid-like reticular stromal networks, an altered cytokine milieu, and the recruitment of regulatory leukocyte populations. In contrast, CCL21-deficient tumors induced antigen-specific immunity. CCL21-mediated immune tolerance was dependent on host rather than tumor expression of the CCL21 receptor, CCR7, and could protect distant, coimplanted CCL21-deficient tumors and even nonsyngeneic allografts from rejection. We suggest that by altering the tumor microenvironment, CCL21-secreting tumors shift the host immune response from immunogenic to tolerogenic, which facilitates tumor progression.

$\mathrm{C}$ ancer fate, including progression, metastasis, and therapy resistance, is largely determined by the interactions between a tumor and host immune cells. Immune cells can recognize tumors by their antigenic profiles, but many tumors manipulate these cells to escape immune surveillance. To accomplish this, tumors can mimic immune signaling pathways that alter the tumor microenvironment to favor the activation of regulatory $\mathrm{T}\left(\mathrm{T}_{\mathrm{reg}}\right)$ cells and suppress effector functions (1-3), driving immunological tolerance and tumor progression.

Here, we examine a mechanism of tumorinduced immune tolerance that bears similarities to the tolerance-maintaining functions of the lymph node (LN) stroma. In the lymph node paracortex, specialized stromal cells called fibroblastic reticular cells (FRCs) secrete the CCR7 ligands CCL21 and CCL19, which guide the interactions between $\mathrm{CCR}^{+} \mathrm{T}$ cells and antigen-presenting cells (APCs) needed for T cell education and priming. Although these events are sufficient to trigger adaptive immunity, they are also necessary for maintaining peripheral tolerance, because $T_{\text {reg }}$ cells require $\mathrm{LN}$ occupancy and CCR7 signaling for their activation and function (4-6), and the loss of CCR7 signaling is associated with spontaneous autoimmunity $(7,8)$. The lymph node stroma itself can also promote deletion of self-reactive cells $(9,10)$ and can help to maintain homeostasis of naïve T cells (11). We recently showed that invasive tumor cells secrete CCL21 (12), and we verified this here in several invasive human tumor lines cultured in three-dimensional conditions (fig. S1A). Given the critical role of CCR7 in both immunity and tolerance, we asked how endogenous tumor CCL21 expression would affect the host immune response.

Aside from recruiting leukocytes and guiding their interactions in the LN, CCL21 is also a main driver of lymphoid tissue formation (13-15), as it attracts $\mathrm{CCR}^{+}$lymphoid tissue inducer (LTi) cells that drive the maturation of lymphoid stroma (16). Notably, expression of CCL19 and CCL21 in nonlymphoid tissues has been correlated with autoimmunity and inflammation, as

Institute of Bioengineering, École Polytechnique Fédérale de Lausanne, 1015 Lausanne, Switzerland.

*These authors contributed equally.

†To whom all correspondence should be addressed. E-mail: melody.swartz@epfl.ch
Fig. 1. CCL21 expression promotes tumor growth that is host CCR7-dependent. (A) CCL21 protein levels (determined by ELISA) in tumor and naïve lymph node lysates 9 days post-implantation (p.i., $n \geq 4$ ). (B) Day 9 p.i. tumor volumes. Multiple $\mathrm{CCL} 21^{\text {low }}$

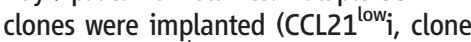
21/217; CCL21 ${ }^{\text {low }}$ ii, clone 21/217 D8;

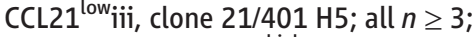
control, $n=22$; $\mathrm{CCL}_{21} 1^{\text {high }}, n=10$ ). Bars show medians \pm SE. (C) Tumor infiltration of $\mathrm{CD}_{4} 5^{+}$leukocytes as determined by flow cytometry, and (D to F) APC subpopulations at day 9 p.i. in wild-type $(n \geq 10)$ and $C C R 7^{-/}$mice $(n=3)$; bars show medians \pm SE. (G) $\mathrm{CCR7}^{+}$leukocyte infiltrates in tumors 9 days p.i. in wild-type $(n \geq 4)$ and $C C R 7^{-/}$mice $(n=3)$. Bars show medians \pm SE. (H) Day 9 p.i. control tumor volumes in wild-type mice treated with CCR7 neutralizing antibodies or control lgG, or in $C C R 7^{-1-}$ mice $(n \geq 3)$. Data represent means \pm SEM. (I) Volumes of single $(n=22)$ and co-implanted tumors $(n \geq 7)$ at day 9 p.i. Data represent means \pm SEM. ${ }^{*} P<$ $0.05,{ }^{* *} P<0.01$ relative to control tumors, and ${ }^{\#} P<0.05$ relative to single implanted $\mathrm{CCL} 21^{\text {low }}$ tumors, one-way analysis of variance (ANOVA) and Bonferroni post-test adjustment.
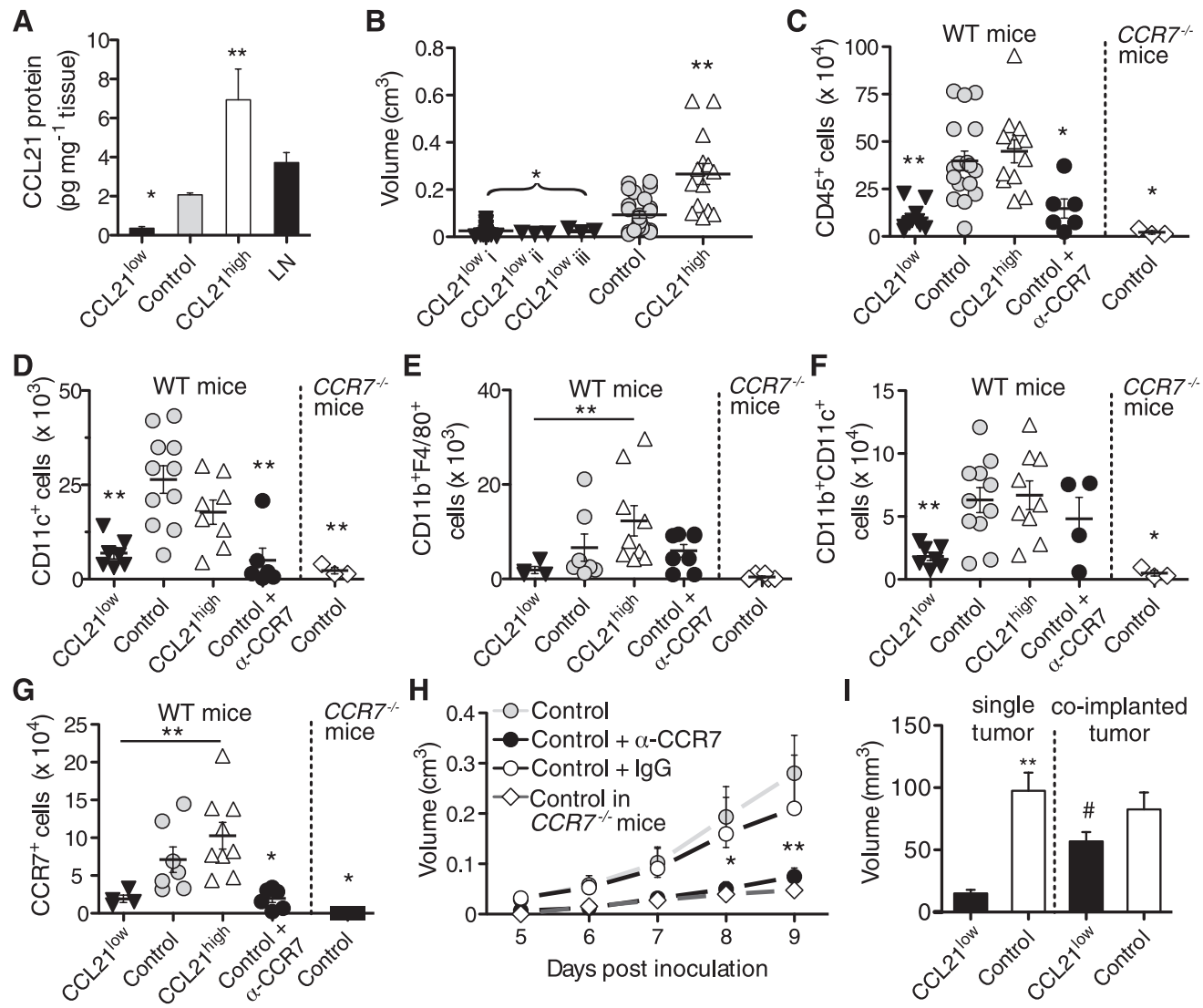

H
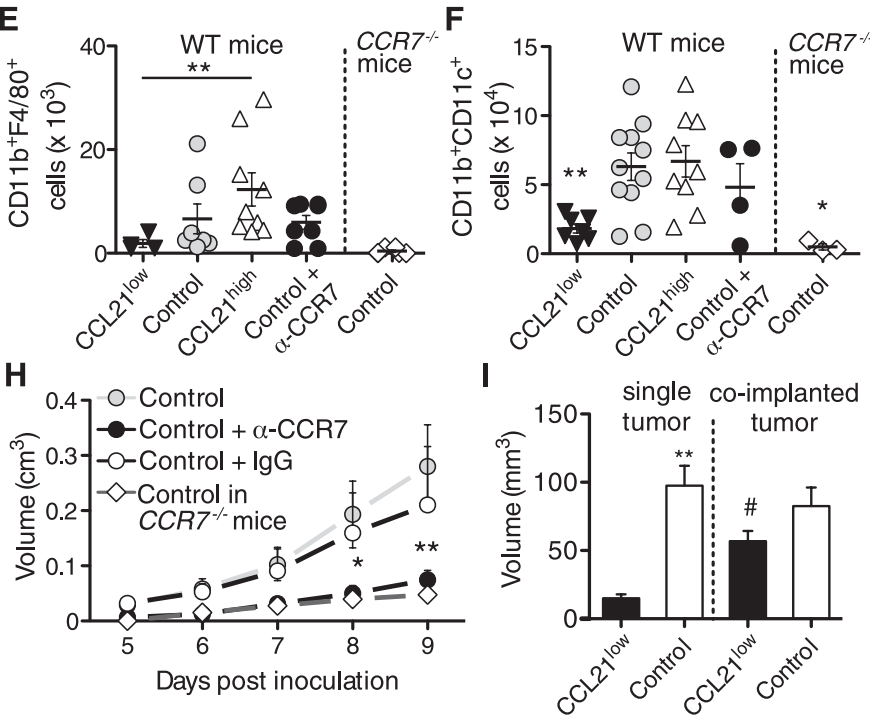
well as immune suppression $(7,15,17)$. Likewise, exogenous CCR7 ligands have been demonstrated to induce both antitumor immunity and tumor immune suppression $(7,18,19)$. Here, in contrast to the studies using exogenous CCR7 ligands, we examine the effects of endogenous melanoma CCL21 expression on tumor fate.

We engineered three stable cell sublines derived from murine B16-F10 melanomas to knockdown endogenous CCL21 secretion by shRNA (CCL21 ${ }^{\text {low }}$ ), to express endogenous amounts (scrambled shRNA control) at amounts comparable to those measured in normal LNs, or to overexpress CCL21 (CCL21 ${ }^{\text {high }}$ ) (20) (Fig. 1A). Surprisingly, when implanted into immune competent syngeneic C57BL/6 mice, CCL2 $1^{\text {high }}$ and control tumors grew significantly larger than CCL $21^{\text {low }}$ tumor clones (Fig. 1B)-even though the CCL21-expressing tumors attracted more $\mathrm{CD} 45^{+}$leukocytes, including APCs and $\mathrm{CCR} 7^{+}$ leukocytes (Fig. 1, C to G, and fig. S1, B to E). Note that $\mathrm{CCR}^{+}$APCs in CCL21-expressing tumors retained the capacity to traffic from the tumor to the draining LNs upon uptake of 0.5 $\mu \mathrm{m}$ fluorescent beads (fig. S2), which suggested that their ability to uptake antigen was not impaired.

Fig. 2. CCL21 expression leads to a tolerogenic tumor microenvironment. (A) Total CD3 $\varepsilon^{+} \mathrm{T}$ cells within $\mathrm{CCL} 21^{\text {low }}$, control, and $\mathrm{CCL} 21^{\text {high }}(n=9)$ tumors $(n \geq 9)$. Bars show medians \pm SE. (B) Number of T cells per unit tumor volume $(n \geq 9)$. Data represent means \pm SEM. (C) Frequency of tyrosinase-related protein-2 peptide SVYDFFVWL (Trp2 $180-188$ )-specific T cells $\left(C D 19^{-}\right.$CD $3 \varepsilon^{+} C D 8 \alpha^{+}$SVYDFFVWL-MHC pentamer $\left.{ }^{+}\right)$ (29) within tumors of wild-type and $C C R 7^{-/-}$mice, as determined by flow cytometry. Bars show medians $\pm \mathrm{SE}$. (D) IFN- $\gamma$ protein levels within tumors as determined by enzyme-linked immunosorbent assay (ELISA) $(n=8)$. Data represent means \pm SEM. (E) Quantification of tumor-infiltrated $\mathrm{T}_{\text {reg }}$ cells within $\mathrm{CCL}_{21}{ }^{\text {low }}$, control, and $\mathrm{CCL} 21^{\text {high }}$ tumors $(n \geq 5)$. Data represent means \pm SEM. (F) Total TGF- $\beta 1$ protein levels within tumors as determined by ELISA $(n \geq 4)$. Data represent means \pm SEM. (G) $\mathrm{CD} 11 \mathrm{C}^{-} \mathrm{CD} 11 \mathrm{~b}^{+} \mathrm{F} 4 / 80^{-} \mathrm{Gr} 1^{\text {high }}$ myeloid-derived suppressor tumor infiltrates $(n \geq 6)$. Bars show medians \pm SE. (H) Intratumoral $\mathrm{T}$ cell populations and $\mathrm{T}_{\text {reg }}$ cells within coimplanted CCL21 ${ }^{\text {low }}$ and control tumors $(n \geq 4)$. Data represent means \pm SEM. (I) Comparison of tumor volumes in wild-type $(n \geq 10)$ and athymic mice (Foxn $1^{n u / n u}, n=$ 4). Bars show medians \pm SE. (J) CD11 $c^{-} C D 11 b^{+} F 4 / 80^{-} G r 1^{\text {high }}$ myeloid-derived suppressor tumor infiltrates in Foxn1 ${ }^{\text {nu/nu }}$ mice $(n=4)$. Bars show medians \pm SE. All data were taken at day 9 p.i. The symbols * or \# indicate $P<0.05$, and double superscripts indicate $P<0.01$ compared with respective control tumors.
These differences in growth could have resulted either from the host response to the tumor or from autocrine effects of CCL21 signaling on tumor cells themselves, because they express CCR7 (fig. S1F). In vitro, however, the three different cell lines proliferated, formed spheroids, and migrated up a gradient of exogenous CCL21 similarly (fig. S1, G to J); furthermore, these behaviors were unaltered by the addition of exogenous CCL21 protein or with CCR7-blocking antibodies, which indicated that the differences seen in growth were dependent on the in vivo environment.

Moreover, tumor growth was host CCR7dependent, because control tumors grew poorly when implanted into CCR7-deficient mice or into wild-type mice treated systemically with CCR7blocking antibodies (Fig. 1, C to H). Therefore, tumor-mediated, CCL21-dependent modulation of the host response, rather than autocrine effects on the tumor itself, was responsible for the differential tumor propagation observed.

To further verify that these growth differences were due to variations in the host immune response to the tumor, rather than to changes incurred on the tumor cells themselves by autocrine CCR7 signaling, we coimplanted CCL2 $1{ }^{\text {low }}$ and control tumors into the same mouse, each on opposite shoulders. In these mice, control tumors could rescue the growth of CCL2 $11^{\text {low }}$ tumors to control levels (Fig. 1I), which further demonstrated that a host response was responsible for the differences in tumor growth seen earlier.

Given this apparent contradiction-increased tumor growth associated with enhanced leukocyte attraction and normal APC trafficking to LNs - we next asked how the secretion of CCL21 could affect interactions between the tumor and its immune cell infiltrates. Upon examining the $\mathrm{T}$ cell populations within the tumors, we found that, although control and CCL2 $1^{\text {high }}$ tumors attracted more T cells overall (Fig. 2A), CCL2 $1^{\text {low }}$ tumors contained higher densities of $\mathrm{T}$ cells (Fig. 2B) and more melanoma antigen [tyrosinase-related protein 2 (Trp2)]-specific CD8 ${ }^{+} \mathrm{T}$ cells (Fig. 2C). This was consistent with increased amounts of interferon- $\gamma$ (IFN- $\gamma)$, interleukin 2 (IL-2), and IL-4 in CCL2 $1^{\text {low }}$ tumors (Fig. 2D and fig. S4, A and B), cytokines that are all associated with cytotoxic $\mathrm{T}$ cell responses and antitumor immunity (1-3). In contrast, control and CCL2 $1^{\text {high }}$ tumors contained more $\mathrm{CD} 4^{+}$ $\mathrm{CD} 25^{+} \mathrm{FoxP}^{+} \mathrm{T}_{\text {reg }}$ cells (Fig. $2 \mathrm{E}$ and fig. S3, A to $C)$ and higher amounts of transforming growth

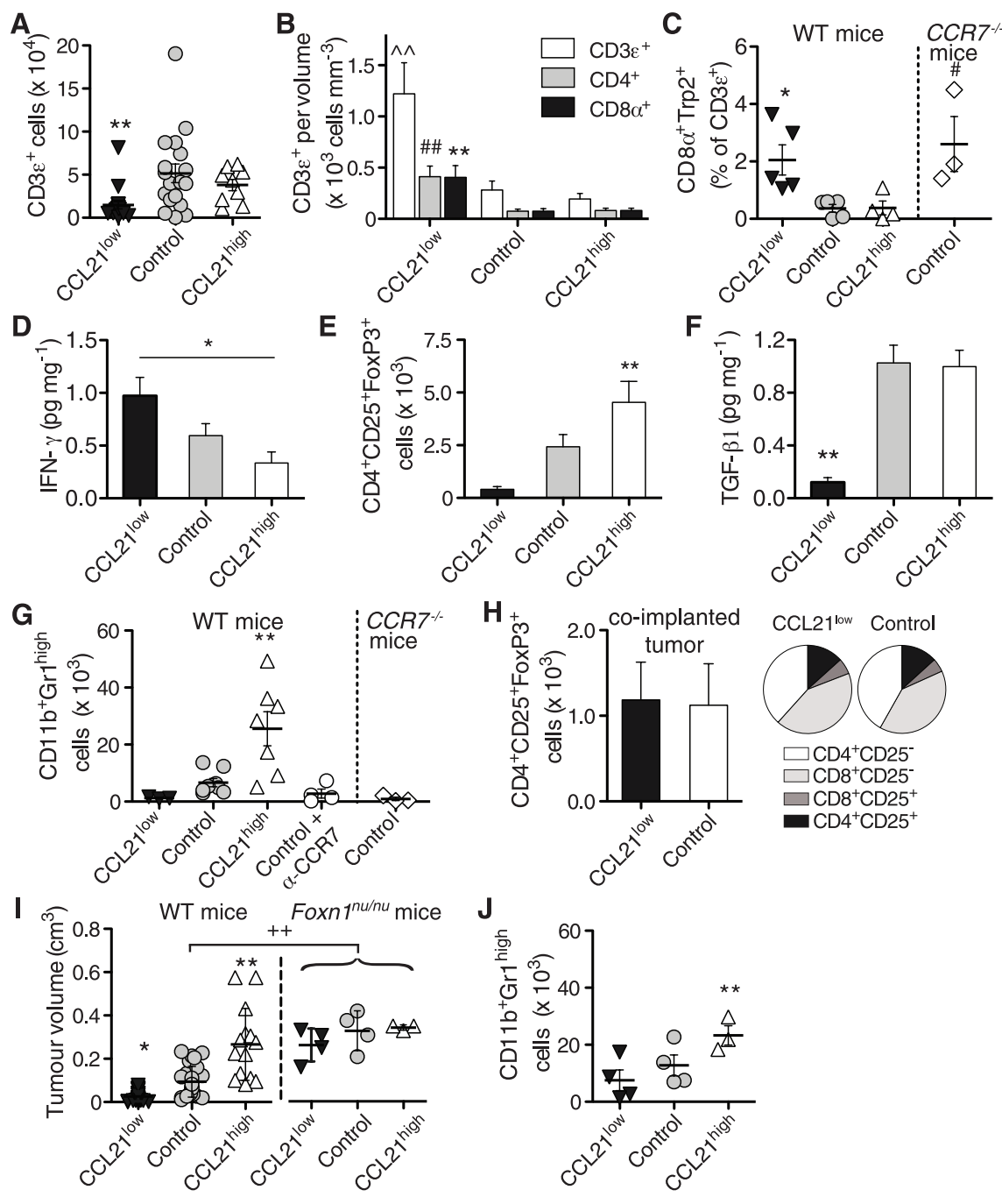


factor- $\beta 1$ (TGF- $\beta 1$ ) (Fig. $2 \mathrm{~F}$ ). TGF- $\beta 1$ is a key regulator of tumor tolerance that suppresses antigen-specific $\mathrm{CD} 8^{+} \mathrm{T}$ cell function, promotes $\mathrm{T}_{\text {reg }}$ cell induction, and shifts the macrophage populations from classically activated (M1) to alternatively activated, protumor (M2) phenotypes $(21,22)$. Furthermore, tumor expression of CCL21 led to enhanced CCR7-dependent attraction of $\mathrm{CD} 11 \mathrm{~b}^{+} \mathrm{CD} 11 \mathrm{c}^{-} \mathrm{F} 4 / 80^{-} \mathrm{Gr}^{\text {high }}$ myeloidderived suppressor cells (MDSCs) that also expressed inducible nitric oxide synthase (Fig. 2G and fig. S4G), a cell type known to drive tumor progression (1-3). Coincident with more MDSCs were higher amounts of the MDSC chemoattractants CCL2 and C5a in CCL21-expressing tumors (fig. S4, D and E). Finally, when implanted into the same mouse but on opposite shoulders, CCL2 $1^{\text {low }}$ and CCL2 $1^{\text {high }}$ tumors (which grew similarly large) (Fig. 1I) displayed similar distributions of $\mathrm{T}$ cell populations (Fig. $2 \mathrm{H}$ ), in- cluding $\mathrm{T}_{\text {reg }}$ cell numbers similar to those seen in CCL $21^{\text {high }}$ tumors grown alone.

The central role of the adaptive immune establishment was further demonstrated by using athymic Foxn $1^{n u / n u}$ mice, which lack T cells and thus cell-mediated immunity. In these mice, CCL2 $1{ }^{\text {low }}$ tumors grew just as large as control and CCL2 $1^{\text {high }}$ tumors (Fig. 2I), despite their impaired recruitment of MDSCs (Fig. 2J).

We next examined the tumor stroma, because peripheral expression of CCL21 can drive lymphoid neogenesis via recruitment of CCR $7^{+} \mathrm{LTi}$ cells $(10,13-15,23)$. In the LN paracortex, FRCs are the major source of CCR7 ligands and are characterized by glycoprotein gp38 and the ERTR7 antigen. In control and CCL2 $1^{\text {high }}$ tumors, we observed FRC networks at the tumor margins that were reminiscent of those in the $\mathrm{LN}$ paracortex (Fig. 3, A and B, and fig. S5). These response in the prevention of CCL2 $1{ }^{\text {low }}$ tumor

tumor stromal networks expressed gp38, which in tumors has been associated with poor prognosis (24). CCL21-expressing tumors, but not CCL2 $1^{\text {low }}$ tumors, also expressed the catabolic enzyme indoleamine 2,3-dioxygenase (IDO) (Fig. 3C), a potent tumor immune suppressor (25), as well as complement receptor 1-related gene/protein y (Crry) (Fig. 3D), a complementregulating protein that helps maintain selftolerance and that can inhibit antitumor immunity (26). Blood vessel density appeared similar in all tumors (Fig. 3E), but some vessels in control and CCL2 $1^{\text {high }}$ tumors also expressed peripheral node addressin (PNAd), which is normally associated with LN high endothelial venules (HEVs) (fig. S5C).

Consistent with these LN-like stromal changes, $\mathrm{CD} 45^{+} \mathrm{CD} 3 \varepsilon^{-} \mathrm{CD} 4^{+} \mathrm{ROR} \gamma \mathrm{t}^{+} \mathrm{LTi}$ cells were preferentially recruited to control and CCL2 $1^{\text {high }}$ tumors in a host CCR7-dependent manner,

$\mathbf{F}$
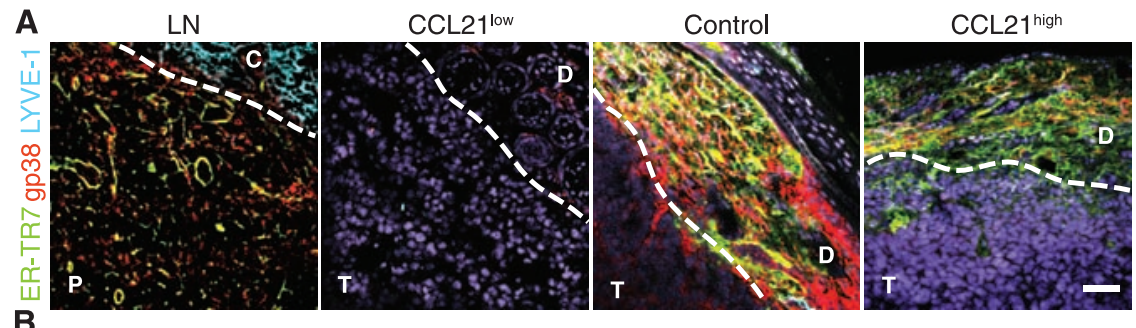

B
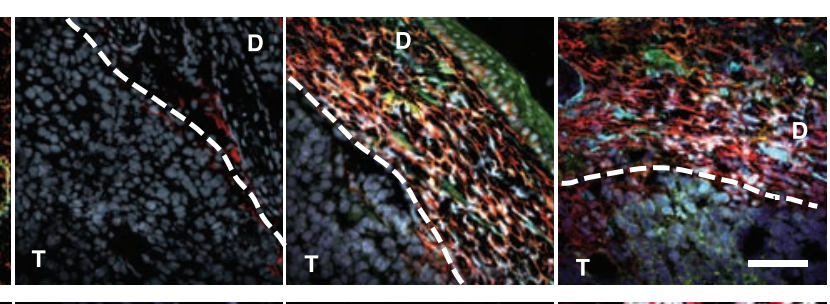

C
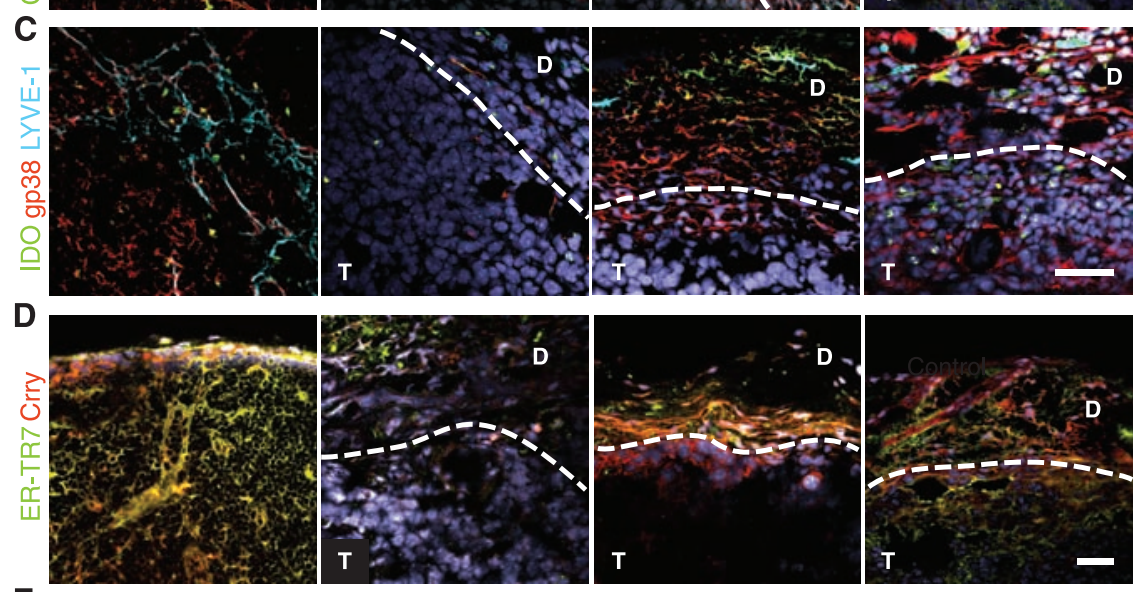

E
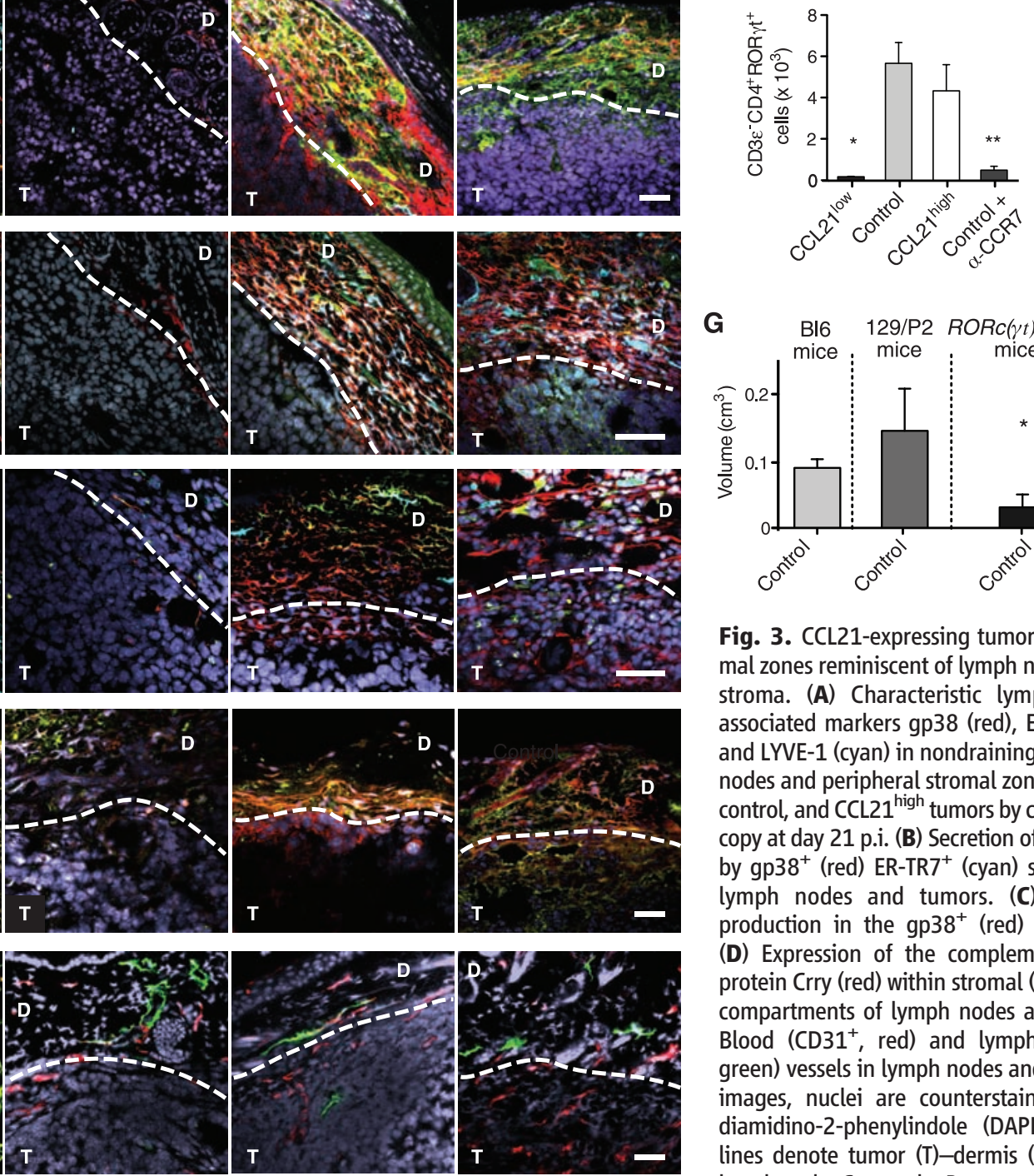

G

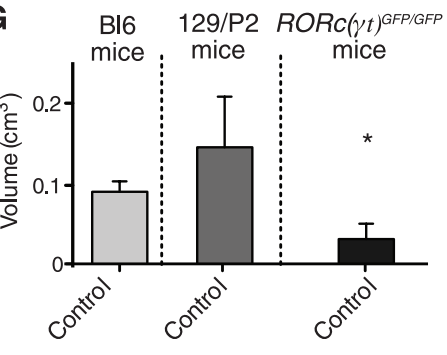

Fig. 3. CCL21-expressing tumors develop stromal zones reminiscent of lymph node paracortex stroma. (A) Characteristic lymphoid stromaassociated markers gp38 (red), ER-TR7 (green), and LYVE-1 (cyan) in nondraining axillary lymph nodes and peripheral stromal zones of CCL21 ${ }^{\text {low }}$, control, and CCL21 $1^{\text {high }}$ tumors by confocal microscopy at day 21 p.i. (B) Secretion of CCL21 (green) by $\mathrm{gp} 8^{+}$(red) ER-TR7 ${ }^{+}$(cyan) stromal cells in lymph nodes and tumors. (C) IDO (green) production in the $\mathrm{gp} 38^{+}$(red) tumor stroma. (D) Expression of the complement regulating protein Crry (red) within stromal (ER-TR7, green) compartments of lymph nodes and tumors. (E) Blood $\left(\mathrm{CD} 31^{+}\right.$, red) and lymphatic (LYVE-1 ${ }^{+}$ green) vessels in lymph nodes and tumors. In all images, nuclei are counterstained with $4^{\prime}, 6^{\prime}$ diamidino-2-phenylindole (DAPI), and dotted lines denote tumor (T)-dermis (D) border. LN, lymph node; C, capsule; $P$, paracortex. Scale bars, $50 \mu \mathrm{m}$. (F) Numbers of $\mathrm{CD} 3 \varepsilon^{-} \mathrm{CD} 4^{+} \mathrm{ROR} \gamma \mathrm{t}^{+} \mathrm{LTi}$ cells detected in tumors from wild-type $(n \geq 3)$ or $C C R T^{-}$mice (control tumors only, $\left.n=4\right)$. Bars show medians \pm SE. (G) Volumes of control tumors day 9 p.i. from wild-type C57/BL6 $(n=22)$, wild-type 129/P2 $(n=5)$, and LTi-deficient $\operatorname{Rorc}(\gamma t)^{G F P / G F P}$ mice $(n=4)$. Data represent means \pm SEM. 

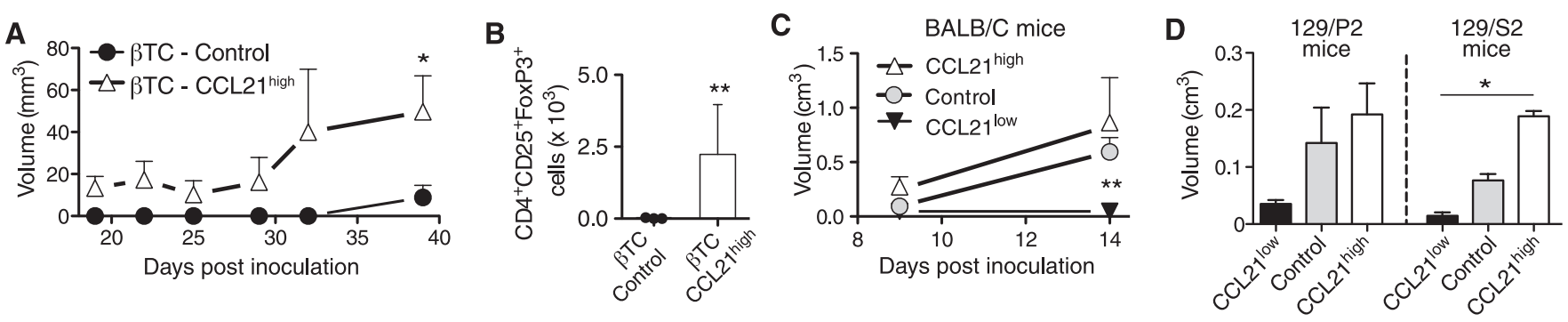

Fig. 4. CCL21 promotes survival of orthotopic and nonsyngeneic tumor allografts. (A) Growth rates for orthotopically implanted control-transfected and CCL21-overexpressing $\beta$ tumor cells ( $\beta T C$-control and $\beta \mathrm{TC}-\mathrm{CCL} 21^{\text {high }} ; n=7$ ). Data represent means \pm SEM. (B) Intratumoral $T_{\text {reg }}$ cells within control $\beta$ tumors. Data represent means \pm SEM. (C) Volumes of $\mathrm{CCL} 21^{\text {low }}$, control, and $\mathrm{CCL} 21^{\text {high }}$

tumors in nonsyngeneic BALB/C recipients at days 9 and 14 p.i. $(n=4)$. Data represent means \pm SEM. (D) Volumes of $C C L 21^{\text {low }}$, control, and $\mathrm{CCL} 21^{\text {high }}$ tumors in nonsyngeneic $129 / \mathrm{P} 2$ and $129 / \mathrm{S} 2$ recipients 9 days p.i. $(n \geq 2)$. Bars show medians $\pm \mathrm{SE}$. ${ }^{*} P<0.05,{ }^{* *} P<0.01$ compared with relevant controls. both in wild-type mice (Fig. 3F and fig. S6A) and $\operatorname{Rorc}(\gamma t)^{+/ G F P}$ mice, which generate GFPexpressing LTi cells (23) (fig. S6B). In contrast, CCL21-enhanced tumor growth was absent in LTi-deficient $\operatorname{Rorc}(\gamma t)^{G F P / G F P}$ mice (Fig. 3G). Therefore, tumor expression of CCL21 was correlated with LTi-cell recruitment, although it is not clear whether LTi-cell recruitment was required for the CCL21-enhanced tumor growth and host immune tolerance observed.

This host tolerogenic response to CCL21secreting tumors could also be demonstrated with another murine tumor cell line, islet beta tumor cells ( $\beta \mathrm{TCs}$ ) (27), which, when transduced to stably overexpress CCL21, grew significantly larger and contained more $\mathrm{T}_{\text {reg }}$ cells than controltransduced counterparts in syngeneic C57BL/6 mice (Fig. 4, A and B). In addition, CCL21 overexpression could even rescue nonsyngeneic allografts, including B16-F10 melanomas implanted into BALB/C,129/P2 and S2 mice (Fig. 4, $\mathrm{C}$ and $\mathrm{D})$. In these cases, we again found that, although CCL2 $1^{\text {low }}$ tumors grew poorly, control and CCL $21^{\text {high }}$ tumors grew robustly.

Taken together, these data suggest that CCL21 secretion by tumors led to a tolerogenic tumor microenvironment with stromal features resembling those of the LN paracortex. Consistent with recent findings that LN stroma itself plays an important role in promoting tolerance to selfantigens (9), we hypothesize that tumor CCL21driven mimicry of the LN stroma helps promote a tolerogenic switch in the host immune response.

Several functions of CCL21 could help to drive the regulatory shift in the $\mathrm{T}$ cell populations that we saw in CCL21-expressing tumors. CCL21 can recruit naïve $T$ cells to peripheral sites $(15,28)$ and can promote their differentiation into $\mathrm{T}_{\text {reg }}$ cells while inducing effector $\mathrm{T}$ cell senescence (7). The coincident development of the specialized stroma in CCL21-expressing tumors might enhance both $\mathrm{T}$ cell trafficking into the tumor and their interactions with APCs or even the stromal cells themselves (9). Such interactions within the regulatory cytokine environment of the tumor may promote $\mathrm{T}_{\text {reg }}$ cell activation and a further shift in the cytokine microenvironment toward one that is less immunogenic.
These changes are consistent with earlier reports that CCR7 signaling is required for the maintenance of peripheral self-tolerance $(4,7,8)$, and with reports demonstrating promotion of deletional tolerance by the lymphoid stroma $(9,10)$. In contrast, CCL21 has also been associated with autoimmunity $(7,15)$, as it drives the formation of lymphocytic infiltrates and tertiary lymphoid structures. Such structures are characterized by B cell follicle formation (14); however, we did not detect $\mathrm{B}$ cell clusters in the tumors examined here (fig. S4F). These conflicting reports (7) emphasize that the timing and context in which $\mathrm{CCR}^{+}$ leukocytes are recruited to the tumor, and the tumor cytokine environment, can modulate the outcome. Furthermore, it has been suggested that CCR7 ligands in the tumor might inhibit allograft rejection by entrapment of the APCs inside the tumor, which prevents them from migrating out to mount an immune response (19), and which our data do not support (fig. S2). Instead, we found CCL21-expressing tumors could prevent rejection of a nonsyngeneic allograft. We propose that, whereas CCL21-secreting tumors attract MDSCs, $T_{\text {reg }}$ cells, and naïve $T$ cells to the tumor microenvironment, it also induces lymphoid-like stroma that can equip the developing tumor with a substrate to promote the induction of $\mathrm{T}_{\text {reg }}$ cells and further guide naïve $T$ cell interactions with APCs, all under a regulatory cytokine milieu (fig. S7). These findings hold therapeutic significance, particularly to tumor vaccine strategies and to emerging antitumor immunotherapies utilizing chemokines (including CCL21 and CCL19) (7) to functionally bias the recruited immune cell infiltrates within the tumor.

\section{References and Notes}

1. A. Ben-Baruch, Cancer Metastasis Rev. 25, 357 (2006).

2. A. Mantovani, P. Allavena, A. Sica, F. Balkwill, Nature 454, 436 (2008).

3. W. Zou, Nat. Rev. Cancer 5, 263 (2005).

4. M. A. Schneider, ]. G. Meingassner, M. Lipp, H. D. Moore, A. Rot, J. Exp. Med. 204, 735 (2007).

5. A. Menning et al., Eur. J. Immunol. 37, 1575 (2007).

6. J. C. Ochando et al., J. Immunol. 174, 6993 (2005).

7. R. Förster, A. C. Davalos-Misslitz, A. Rot, Nat. Rev. Immunol. 8, 362 (2008)
8. A. C. Davalos-Misslitz et al., Eur. J. Immunol. 37, 613 (2007).

9. J. W. Lee et al., Nat. Immunol. 8, 181 (2007).

10. S. N. Mueller, R. N. Germain, Nat. Rev. Immunol. 9, 618 (2009).

11. A. Link et al., Nat. Immunol. 8, 1255 (2007).

12. J. D. Shields et al., Cancer Cell 11, 526 (2007)

13. T. D. Randall, D. M. Carragher, ]. Rangel-Moreno, Annu. Rev. Immunol. 26, 627 (2008).

14. D. L. Drayton, S. Liao, R. H. Mounzer, N. H. Ruddle, Nat. Immunol. 7, 344 (2006).

15. W. Weninger et al., J. Immunol. 170, 4638 (2003).

16. G. Eberl, D. R. Littman, Immunol. Rev. 195, 81 (2003).

17. L. Peduto et al., J. Immunol. 182, 5789 (2009).

18. S. Krautwald et al., Immunology 112, 301 (2004).

19. E. Ziegler et al., J. Am. Soc. Nephrol. 17, 2521 (2006)

20. Materials and methods are available as supporting material on Science Online.

21. B. Bierie, H. L. Moses, Nat. Rev. Cancer 6, 506 (2006).

22. A. Sica et al., Semin. Cancer Biol. 18, 349 (2008).

23. G. Eberl et al., Nat. Immunol. 5, 64 (2004).

24. A. Kawase et al., Int. J. Cancer 123, 1053 (2008)

25. G. C. Prendergast, Oncogene 27, 3889 (2008).

26. J. C. Varela et al., Cancer Res. 68, 6734 (2008).

27. J. A. Joyce et al., Cancer Cell 4, 393 (2003).

28. M. R. Britschgi, A. Link, T. K. Lissandrin, S. A. Luther, J. Immunol. 181, 7681 (2008).

29. Single-letter abbreviations for the amino acid residues are as follows: $A, A l a ; C, C y s ; D, A s p ; E$, Glu; F, Phe; G, Gly; H, His; I, Ile; K, Lys; L, Leu; M, Met; N, Asn; P, Pro; Q, Gln; R, Arg; S, Ser; T, Thr; V, Val; W, Trp; and Y, Tyr.

30. We thank G. Eberl for $\operatorname{Rorc}(\gamma t)^{+/ G F P}$ and $\operatorname{Rorc}(\gamma t)^{\text {GFP/GFP }}$ mice, S. Luther for $C C R 7^{-/}$mice, D. Hanahan for $\beta$ tumor cells, and D. Trono for lentiviral vector plasmids; S. Pradervand, A. Paillusson, D. Foretay, M. Pasquier, V. Borel, B. Dixon, and A. Jimenez for technical assistance; and J. Hubbell and G. Eberl for critical reading of the manuscript. Funding was provided by the Swiss Cancer League, the Swiss National Science Foundation, the European Research Council, and the U.S. Department of Defense Breast Cancer Research Program to M.A.S. The authors (except J.M.R.) have filed a patent application on the use of CCL21 in immune modulation.

\section{Supporting Online Material}

www.sciencemag.org/cgi/content/full/science.1185837/DC1 Materials and Methods

Figs. S1 to S7

11 December 2009; accepted 16 March 2010 Published online 25 March 2010;

$10.1126 /$ science. 1185837

Include this information when citing this paper. 